\title{
In Situ Observation of Structural and Morphological Changes in Nanometer-sized Silver Particles on Graphite during Sublimation
}

\author{
H. Mori and J. -G. Lee
}

Research Center for Ultra High Voltage Electron Microscopy, Osaka University, 7-1, Mihogaoka, Ibaraki, Osaka 567-0047, Japan

Recent developments in high resolution electron microscopy (HREM) have greatly contributed to the understanding of the origin of the unique structures and properties of nanometer-sized particles [1-3]. It is of significance to directly confirm whether sublimation would take place from a nanometer-sized particle on a substrate and continue until whole the particle would disappear or a particle would first undergo a solid-to-liquid (i.e., melting) transition and then undergo a liquid-to-gas transition until all the constituent atoms of the particle would evaporate. It is also of interest to examine the change in morphology of a particle during such a solid-to-gas (or liquid-to-gas) transition as a function of particle size. Based upon this premise, in the present work, structural and morphological changes in nanometer-sized silver particles associated with sublimation-induced size reduction, have been studied by in-situ HREM.

Nanometer-sized silver particles were produced and observed in the same vacuum chamber (the base pressure was below $7 \times 10^{-7} \mathrm{~Pa}$ ) of a Hitachi HF-2000 type $200 \mathrm{kV}$ HREM without exposing particles to any undesired atmosphere using a side-entry holder which was equipped with an evaporator at its tip. The evaporator consisted of two spiral-shaped tungsten filaments. One of the filaments was attached with a flake of graphite (supporting substrate) and the other with silver (source material). Prior to experiment, the flake was baked at around $1100 \mathrm{~K}$ for $60 \mathrm{~s}$ to obtain a cleaned surface. After the graphite substrate was cooled down to around $600 \mathrm{~K}$, then silver was evaporated from the second filament onto the substrate kept at the same temperature, and nanometer-sized silver particles were produced on the edge of the graphite flake. After being formed on the graphite substrate, silver particles were once cooled down to room temperature. The particles were then subjected to in-situ annealing experiments in the microscope; the particles were heated slowly up to $950 \mathrm{~K}$ and kept at the temperature, and any phase transitions and morphological changes which may occur in the particles were monitored in-situ.

A typical crystalline-to-gas transition in a nanometer-sized silver particle on the graphite substrate kept at $950 \mathrm{~K}$ is depicted in Fig. 1. The number inserted in each micrograph indicates relative time in seconds. It is seen that the crystalline silver particle is oriented with the (111) face parallel to the (0001) face of graphite substrate, which is consistent with the orientation relationship reported in the literature [4]. As shown in Fig. 1(a)-(f), it is obvious that evaporation of silver atoms from an approximately 9-nm-sized crystalline silver particle occurred (that is, sublimation occurred) at 950K, which eventually led to the complete disappearance of the silver particle (Fig. 1(f)). It should be noted that no crystalline-to-liquid transition took place during this process. The morphology of silver particles on the graphite substrate was also studied as a function of particle size. As a quantitative measure of the particle morphology, the height-to-width ratio (as for definition, see the inset in Fig. 2) was taken. The ratio was continuously measured during the sublimation-induced shrinkage of the silver particle. A typical result of the size dependence of the height-to-width ratio is shown in Fig. 2. The data have been taken from the silver particle depicted in Fig. 1. In Fig. 2, it is clear that the 
height-to-width ratio is almost constant, 0.65, during the sublimation-induced shrinkage of the particle. Based upon these results, the behavior of nanometer-sized silver particles supported on the graphite substrate has been discussed [5].

\section{References}

[1] J. Spence, Materials Today 5 (2002) 20.

[2] J. -G. Lee and H. Mori, Phys. Rev. Lett. 93 (2004) 235501.

[3] J. -G. Lee, H. Mori, and H. Yasuda, J. Mater. Res. 20 (2005) 1708.

[4] O. Dezellus and N. Eustathopoulos, Scripta Materialia 40 (1999) 1283.

[5] J. -G. Lee. J. Lee, T. Tanaka, and H. Mori, Phys. Rev. Lett. (2006) in press.

[6] This work was supported by "Priority Assistance of the Formation of Worldwide Renowned Centers of Research - The 21st Century COE Program (Project: Center of Excellence for Advanced Structural and Functional Materials Design)" from the Ministry of Education, Sports, Culture, Science and Technology of Japan. A part of the present work was supported by the Ministry of Education, Sports, Culture, Science and Technology of Japan under Grant-in-Aids for Scientific Research (Grant No. 15074213 and 17206064).
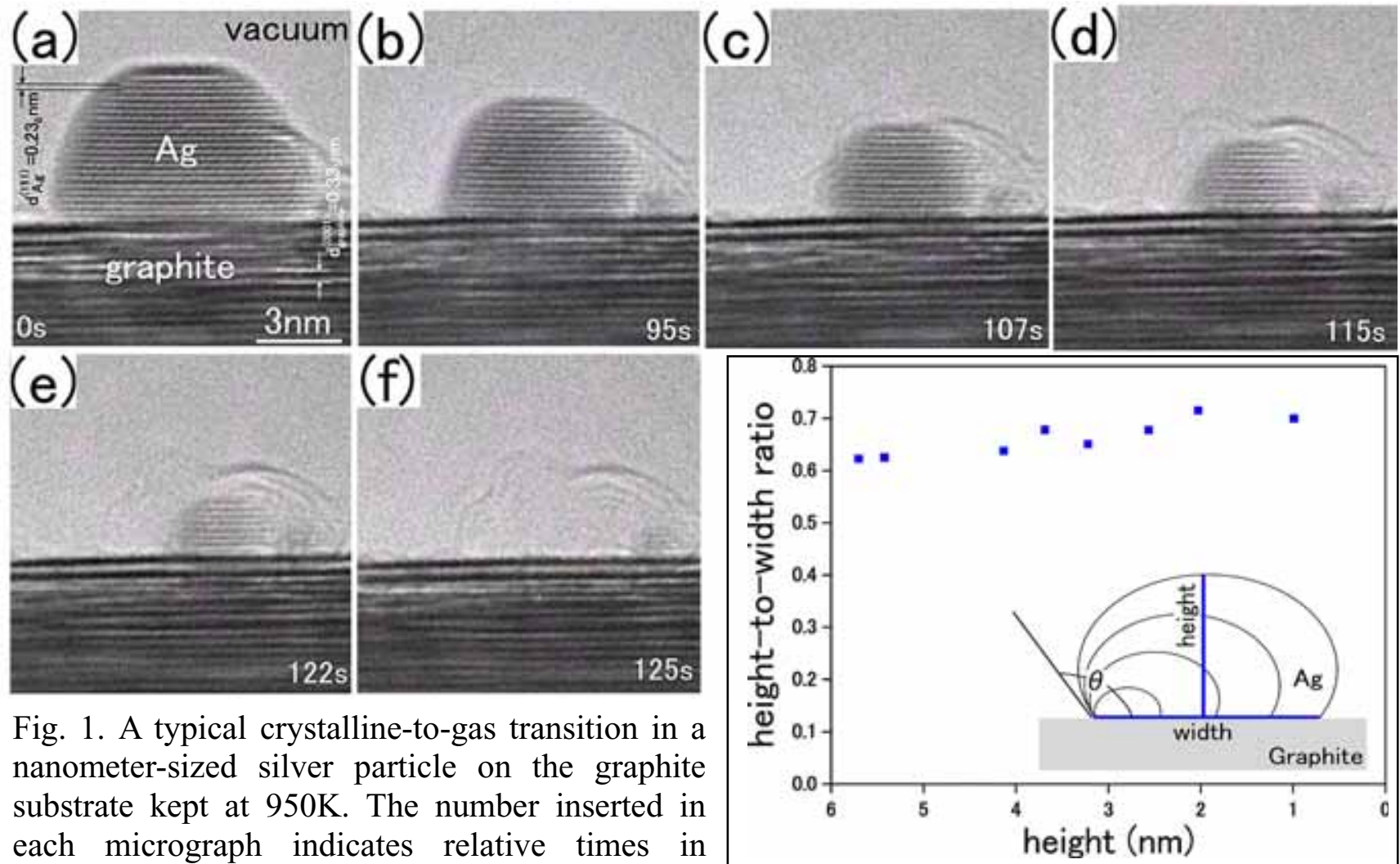

Fig. 1. A typical crystalline-to-gas transition in a nanometer-sized silver particle on the graphite substrate kept at $950 \mathrm{~K}$. The number inserted in each micrograph indicates relative times in seconds. Remarkable sublimation of silver atoms occurred from a crystalline silver particle at $950 \mathrm{~K}$ under the vacuum of $7 \times 10^{-7} \mathrm{~Pa}$ (Fig. (a) to (e)) and it continued until whole the crystalline silver particle disappeared (Fig. (f)). During this process, no melting of the silver particle occurred.

Fig. 2. Size dependence of the height-to-width ratio of a silver particle on graphite at $950 \mathrm{~K}$. The height-to-width ratio was almost constant, 0.65, (or the ratio seems to even slightly increase) during the sublimation-induced shrinkage of the silver particle. 